\title{
Linear Transformations of Euclidean Topological Spaces. Part II
}

\author{
Karol Pąk \\ Institute of Informatics \\ University of Białystok \\ Poland
}

Summary. We prove a number of theorems concerning various notions used in the theory of continuity of barycentric coordinates.

MML identifier: MATRTOP2, version: $\underline{7.11 .07 \quad 4.156 .1112}$

The papers [2], [9], [4], [5], [6], [14], [10], [25], [13], [16], [3], [7], [12], [1], [24], [15], [21], [23], [19], [17], [8], [11], [22], [20], and [18] provide the terminology and notation for this paper.

\section{Correspondence Between Euclidean Topological Space and VECTOR SPACE OVER $\mathbb{R}_{\mathrm{F}}$}

For simplicity, we follow the rules: $X$ denotes a set, $n, m, k$ denote natural numbers, $K$ denotes a field, $f$ denotes an $n$-element real-valued finite sequence, and $M$ denotes a matrix over $\mathbb{R}_{\mathrm{F}}$ of dimension $n \times m$.

One can prove the following propositions:

(1) $X$ is a linear combination of the $n$-dimension vector space over $\mathbb{R}_{\mathrm{F}}$ if and only if $X$ is a linear combination of $\mathcal{E}_{\mathrm{T}}^{n}$.

(2) Let $L_{2}$ be a linear combination of the $n$-dimension vector space over $\mathbb{R}_{F}$ and $L_{1}$ be a linear combination of $\mathcal{E}_{\mathrm{T}}^{n}$. If $L_{1}=L_{2}$, then the support of $L_{1}=$ the support of $L_{2}$.

(3) Let $F$ be a finite sequence of elements of $\mathcal{E}_{\mathrm{T}}^{n}, f_{1}$ be a function from $\mathcal{E}_{\mathrm{T}}^{n}$ into $\mathbb{R}, F_{1}$ be a finite sequence of elements of the $n$-dimension vector space over $\mathbb{R}_{\mathrm{F}}$, and $f_{2}$ be a function from the $n$-dimension vector space over $\mathbb{R}_{\mathrm{F}}$ into $\mathbb{R}_{\mathrm{F}}$. If $f_{1}=f_{2}$ and $F=F_{1}$, then $f_{1} F=f_{2} F_{1}$. 
(4) Let $F$ be a finite sequence of elements of $\mathcal{E}_{\mathrm{T}}^{n}$ and $F_{1}$ be a finite sequence of elements of the $n$-dimension vector space over $\mathbb{R}_{\mathrm{F}}$. If $F_{1}=F$, then $\sum F=\sum F_{1}$.

(5) Let $L_{2}$ be a linear combination of the $n$-dimension vector space over $\mathbb{R}_{\mathrm{F}}$ and $L_{1}$ be a linear combination of $\mathcal{E}_{\mathrm{T}}^{n}$. If $L_{1}=L_{2}$, then $\sum L_{1}=\sum L_{2}$.

(6) Let $A_{2}$ be a subset of the $n$-dimension vector space over $\mathbb{R}_{\mathrm{F}}$ and $A_{1}$ be a subset of $\mathcal{E}_{\mathrm{T}}^{n}$. If $A_{2}=A_{1}$, then $\Omega_{\operatorname{Lin}\left(A_{1}\right)}=\Omega_{\operatorname{Lin}\left(A_{2}\right)}$.

(7) Let $A_{2}$ be a subset of the $n$-dimension vector space over $\mathbb{R}_{\mathrm{F}}$ and $A_{1}$ be a subset of $\mathcal{E}_{\mathrm{T}}^{n}$. Suppose $A_{2}=A_{1}$. Then $A_{2}$ is linearly independent if and only if $A_{1}$ is linearly independent.

(8) Let $V$ be a vector space over $K, W$ be a subspace of $V$, and $L$ be a linear combination of $V$. Then $L /$ the carrier of $W$ is a linear combination of $W$.

(9) Let $V$ be a vector space over $K, A$ be a linearly independent subset of $V$, and $L_{3}, L_{4}$ be linear combinations of $V$. Suppose the support of $L_{3} \subseteq A$ and the support of $L_{4} \subseteq A$ and $\sum L_{3}=\sum L_{4}$. Then $L_{3}=L_{4}$.

(10) Let $V$ be a real linear space, $W$ be a subspace of $V$, and $L$ be a linear combination of $V$. Then $L /$ the carrier of $W$ is a linear combination of $W$.

(11) Let $U$ be a subspace of the $n$-dimension vector space over $\mathbb{R}_{\mathrm{F}}$ and $W$ be a subspace of $\mathcal{E}_{\mathrm{T}}^{n}$. Suppose $\Omega_{U}=\Omega_{W}$. Then $X$ is a linear combination of $U$ if and only if $X$ is a linear combination of $W$.

(12) Let $U$ be a subspace of the $n$-dimension vector space over $\mathbb{R}_{\mathrm{F}}, W$ be a subspace of $\mathcal{E}_{\mathrm{T}}^{n}, L_{5}$ be a linear combination of $U$, and $L_{6}$ be a linear combination of $W$. If $L_{5}=L_{6}$, then the support of $L_{5}=$ the support of $L_{6}$ and $\sum L_{5}=\sum L_{6}$.

Let us consider $m, K$ and let $A$ be a subset of the $m$-dimension vector space over $K$. Note that $\operatorname{Lin}(A)$ is finite dimensional.

\section{Correspondence Between the Mx2Tran Operator and Decomposition of a Vector in Basis}

The following propositions are true:

(13) If $\operatorname{rk}(M)=n$, then $M$ is an ordered basis of $\operatorname{Lin}(\operatorname{lines}(M))$.

(14) Let $V, W$ be vector spaces over $K, T$ be a linear transformation from $V$ to $W, A$ be a subset of $V$, and $L$ be a linear combination of $A$. If $T\lceil A$ is one-to-one, then $T\left(\sum L\right)=\sum\left(T^{@} L\right)$.

(15) Let $S$ be a subset of $\operatorname{Seg} n$. Suppose $M\lceil S$ is one-to-one and $\operatorname{rng}(M\lceil S)=$ lines $(M)$. Then there exists a linear combination $L$ of $\operatorname{lines}(M)$ such that $\sum L=(\operatorname{Mx} 2 \operatorname{Tran} M)(f)$ and for every $k$ such that $k \in S$ holds $L(\operatorname{Line}(M, k))=\sum \operatorname{Seq}\left(f\left\lceil M^{-1}(\{\operatorname{Line}(M, k)\})\right)\right.$. 
(16) Suppose $M$ is without repeated line. Then there exists a linear combination $L$ of lines $(M)$ such that $\sum L=(\operatorname{Mx} 2 \operatorname{Tran} M)(f)$ and for every $k$ such that $k \in \operatorname{dom} f$ holds $L(\operatorname{Line}(M, k))=f(k)$.

(17) For every ordered basis $B$ of $\operatorname{Lin}(\operatorname{lines}(M))$ such that $B=M$ and for every element $M_{1}$ of $\operatorname{Lin}(\operatorname{lines}(M))$ such that $M_{1}=(\operatorname{Mx} 2 \operatorname{Tran} M)(f)$ holds $M_{1} \rightarrow B=f$.

(18) $\operatorname{rng} M x 2 \operatorname{Tran} M=\Omega_{\operatorname{Lin}(\operatorname{lines}(M))}$.

(19) Let $F$ be a one-to-one finite sequence of elements of $\mathcal{E}_{\mathrm{T}}^{n}$. Suppose $\operatorname{rng} F$ is linearly independent. Then there exists a square matrix $M$ over $\mathbb{R}_{\mathrm{F}}$ of dimension $n$ such that $M$ is invertible and $M\lceil$ len $F=F$.

(20) Let $B$ be an ordered basis of the $n$-dimension vector space over $\mathbb{R}_{\mathrm{F}}$. If $B=\operatorname{MX} 2 \operatorname{FinS}\left(I_{\mathbb{R}_{F}}^{n \times n}\right)$, then $f \in \operatorname{Lin}(\operatorname{rng}(B \nmid k))$ iff $f=\left(f\lceil k)^{\frown}\left(\left(n-^{\prime} k\right) \mapsto\right.\right.$ $0)$.

(21) Let $F$ be a one-to-one finite sequence of elements of $\mathcal{E}_{\mathrm{T}}^{n}$. Suppose $\operatorname{rng} F$ is linearly independent. Let $B$ be an ordered basis of the $n$-dimension vector space over $\mathbb{R}_{\mathrm{F}}$. Suppose $B=\operatorname{MX} 2 \operatorname{FinS}\left(I_{\mathbb{R}_{\mathrm{F}}}^{n \times n}\right)$. Let $M$ be a square matrix over $\mathbb{R}_{\mathrm{F}}$ of dimension $n$. If $M$ is invertible and $M \uparrow \operatorname{len} F=F$, then $(\operatorname{Mx} 2 \operatorname{Tran} M)^{\circ}\left(\Omega_{\operatorname{Lin}(\operatorname{rng}(B \nmid \operatorname{len} F))}\right)=\Omega_{\operatorname{Lin}(\operatorname{rng} F)}$.

(22) Let $A, B$ be linearly independent subsets of $\mathcal{E}_{\mathrm{T}}^{n}$. Suppose $\overline{\bar{A}}=\overline{\bar{B}}$. Then there exists a square matrix $M$ over $\mathbb{R}_{\mathrm{F}}$ of dimension $n$ such that $M$ is invertible and $(\operatorname{Mx} 2 \operatorname{Tran} M)^{\circ}\left(\Omega_{\operatorname{Lin}(A)}\right)=\Omega_{\operatorname{Lin}(B)}$.

\section{Preservation of Linear and Affine Independence of Vectors by THE Mx2Tran Operator}

The following propositions are true:

(23) For every linearly independent subset $A$ of $\mathcal{E}_{\mathrm{T}}^{n}$ such that $\operatorname{rk}(M)=n$ holds $(\mathrm{Mx} 2 \operatorname{Tran} M)^{\circ} A$ is linearly independent.

(24) For every affinely independent subset $A$ of $\mathcal{E}_{\mathrm{T}}^{n}$ such that $\operatorname{rk}(M)=n$ holds $(\operatorname{Mx} 2 \operatorname{Tran} M)^{\circ} A$ is affinely independent.

(25) Let $A$ be an affinely independent subset of $\mathcal{E}_{\mathrm{T}}^{n}$. Suppose $\operatorname{rk}(M)=n$. Let $v$ be an element of $\mathcal{E}_{\mathrm{T}}^{n}$. If $v \in \operatorname{Affin} A$, then $(\operatorname{Mx} 2 \operatorname{Tran} M)(v) \in$ $\operatorname{Affin}\left((\operatorname{Mx} 2 \operatorname{Tran} M)^{\circ} A\right)$ and for every $f$ holds $(v \rightarrow A)(f)=$ $\left((\mathrm{Mx} 2 \operatorname{Tran} M)(v) \rightarrow(\mathrm{Mx} 2 \operatorname{Tran} M)^{\circ} A\right)((\mathrm{Mx} 2 \operatorname{Tran} M)(f))$.

(26) For every linearly independent subset $A$ of $\mathcal{E}_{\mathrm{T}}^{m}$ such that $\operatorname{rk}(M)=n$ holds $(\mathrm{Mx} 2 \operatorname{Tran} M)^{-1}(A)$ is linearly independent.

(27) For every affinely independent subset $A$ of $\mathcal{E}_{\mathrm{T}}^{m}$ such that $\operatorname{rk}(M)=n$ holds $(\mathrm{Mx} 2 \operatorname{Tran} M)^{-1}(A)$ is affinely independent. 


\section{REFERENCES}

[1] Jesse Alama. The rank+nullity theorem. Formalized Mathematics, 15(3):137-142, 2007, doi:10.2478/v10037-007-0015-6.

[2] Grzegorz Bancerek. Cardinal numbers. Formalized Mathematics, 1(2):377-382, 1990.

[3] Grzegorz Bancerek. The fundamental properties of natural numbers. Formalized Mathematics, 1(1):41-46, 1990.

[4] Grzegorz Bancerek and Krzysztof Hryniewiecki. Segments of natural numbers and finite sequences. Formalized Mathematics, 1(1):107-114, 1990.

[5] Czesław Byliński. Functions and their basic properties. Formalized Mathematics, 1(1):5565, 1990.

[6] Czesław Byliński. Functions from a set to a set. Formalized Mathematics, 1(1):153-164, 1990.

[7] Czesław Byliński. Partial functions. Formalized Mathematics, 1(2):357-367, 1990.

[8] Czesław Byliński. The sum and product of finite sequences of real numbers. Formalized Mathematics, 1(4):661-668, 1990.

[9] Agata Darmochwał. The Euclidean space. Formalized Mathematics, 2(4):599-603, 1991.

[10] Katarzyna Jankowska. Matrices. Abelian group of matrices. Formalized Mathematics, 2(4):475-480, 1991.

[11] Eugeniusz Kusak, Wojciech Leończuk, and Michał Muzalewski. Abelian groups, fields and vector spaces. Formalized Mathematics, 1(2):335-342, 1990.

[12] Anna Lango and Grzegorz Bancerek. Product of families of groups and vector spaces. Formalized Mathematics, 3(2):235-240, 1992.

[13] Robert Milewski. Associated matrix of linear map. Formalized Mathematics, 5(3):339345, 1996.

[14] Karol Pąk. Basic properties of the rank of matrices over a field. Formalized Mathematics, 15(4):199-211, 2007, doi:10.2478/v10037-007-0024-5.

[15] Karol Pąk. Affine independence in vector spaces. Formalized Mathematics, 18(1):87-93, 2010, doi: 10.2478/v10037-010-0012-z.

[16] Karol Pąk. Linear transformations of Euclidean topological spaces. Formalized Mathematics, 19(2):103-108, 2011, doi: 10.2478/v10037-011-0016-3.

[17] Wojciech A. Trybulec. Basis of real linear space. Formalized Mathematics, 1(5):847-850, 1990.

[18] Wojciech A. Trybulec. Basis of vector space. Formalized Mathematics, 1(5):883-885, 1990.

[19] Wojciech A. Trybulec. Linear combinations in real linear space. Formalized Mathematics, $1(3): 581-588,1990$

[20] Wojciech A. Trybulec. Linear combinations in vector space. Formalized Mathematics, $1(5): 877-882,1990$.

[21] Wojciech A. Trybulec. Subspaces and cosets of subspaces in real linear space. Formalized Mathematics, 1(2):297-301, 1990.

[22] Wojciech A. Trybulec. Subspaces and cosets of subspaces in vector space. Formalized Mathematics, 1(5):865-870, 1990.

[23] Wojciech A. Trybulec. Vectors in real linear space. Formalized Mathematics, 1(2):291-296, 1990.

[24] Edmund Woronowicz. Relations defined on sets. Formalized Mathematics, 1(1):181-186, 1990.

[25] Xiaopeng Yue, Xiquan Liang, and Zhongpin Sun. Some properties of some special matrices. Formalized Mathematics, 13(4):541-547, 2005.

Received October 26, 2010 